
internationales

vol. $19-n^{\circ} 3 \mid 2003$

Moyen-Orient : mutations récentes d'un carrefour migratoire

\title{
Golfe arabo-persique : un système migratoire de plus en plus tourné vers l'Asie
}

Marc Lavergne

\section{(2) OpenEdition \\ Journals}

Édition électronique

URL : https://journals.openedition.org/remi/2689

DOI : $10.4000 /$ remi.2689

ISSN : $1777-5418$

Éditeur

Université de Poitiers

Édition imprimée

Date de publication : 12 décembre 2003

Pagination : 229-241

ISBN : 2-911627-35-0

ISSN : 0765-0752

Référence électronique

Marc Lavergne, « Golfe arabo-persique : un système migratoire de plus en plus tourné vers l'Asie », Revue européenne des migrations internationales [En ligne], vol. 19 - n³ | 2003, mis en ligne le 09 juin 2006, consulté le 14 avril 2022. URL : http://journals.openedition.org/remi/2689 ; DOI : https://doi.org/ 10.4000/remi.2689

Ce document a été généré automatiquement le 14 avril 2022.

(c) Université de Poitiers 


\title{
Golfe arabo-persique : un système migratoire de plus en plus tourné vers l'Asie
}

\author{
Marc Lavergne
}

1 Depuis la mise en exploitation de leurs ressources en hydrocarbures à partir des années quarante, les pays du Golfe ont attiré une main-d'œuvre étrangère de plus en plus nombreuse. Il y avait déjà, d'après les chiffres de l'OIT, environ 7 millions d'étrangers dans les six pays membres du Conseil de Coopération du Golfe en 1995. Ils représentaient environ $30 \%$ de la population en Arabie Saoudite, $60 \%$ au Koweït, $30 \%$ à Bahreïn, $80 \%$ au Qatar et aux Émirats Arabes Unis, et $25 \%$ en Oman ${ }^{1}$. Encore ces chiffres sont-ils des estimations prises dans des fourchettes basses qui ne tiennent pas compte des immigrants illégaux. Pour les pays d'accueil, la présence de cette masse de population étrangère a des conséquences multiformes; mais il en va de même pour les pays et les régions d'origine des migrants, dont la vie et la situation économique et sociale sont très affectées par cet appel de main-d'œuvre et par les flux d'argent qu'il engendre en retour. L'étude de cette immigration n'a été envisagée jusqu'à présent, pour l'essentiel, que de manière globale, et surtout du point de vue de ses implications économiques et financières. Mais elle devrait constituer un objet de recherches pour les sciences humaines et sociales, pour le droit et la science politique, tout autant que pour l'économie de la région.

2 Le nombre des migrants fluctue dans le temps et dans l'espace : il suit d'assez près les variations du cours du pétrole, c'est-à-dire qu'en période de refroidissement de la croissance, le flux d'entrants diminue et une partie des migrants rentre chez eux, soit volontairement à l'expiration de leur contrat, soit victimes d'expulsions menées par les autorités. Mais la reprise appelle de nouveau un retour de migrants, tant l'économie est dépendante de leur travail, dans tous les secteurs.

De plus leur présence n'est pas répartie de manière uniforme : elle correspond aux pôles d'activité économique, d'une part, et d'autre part elle est concentrée là où se trouve concentrée la richesse; c'est ainsi que la présence des étrangers est bien 
moindre dans les Émirats du Nord ${ }^{2}$, dépourvus de ressources pétrolières que dans les Émirats phares de la Fédération des Émirats Arabes Unis que sont Abou Dhabi et Doubaï; de même, en Oman, la majorité d'entre eux sont concentrés dans l'aire métropolitaine de Mascate, la capitale, tandis qu'ils sont très minoritaires dans les villes et les villages de l'intérieur's

Cette immigration est composite. On y distingue, dans une approche heuristique à la fois diachronique et analytique, trois grandes provenances : l'immigration venue de la rive nord du Golfe, celle issue du monde arabe et celle venue du sous-continent indien et d'Extrême-Orient.

L'immigration venue de la rive nord du Golfe : l'héritage de relations millénaires

5 La rive sud du Golfe persique entretient des relations millénaires avec la rive nord, aujourd'hui la République islamique d'Iran, et le Golfe persique est de ce fait une Méditerranée par les échanges commerciaux et humains qui relient les deux rives. La rive nord, façade d'un empire perse de haute civilisation, a d'ailleurs polarisé, jusqu'à la découverte du pétrole, les flux de la rive sud, modeste chapelet d'oasis et de villages de pêcheurs, adossés au désert d'Arabie ${ }^{4}$ : des groupes venus de la rive nord ont fait souche au fil des siècles sur la rive sud et vice-versa, d'autres ont même opéré des va et vient en fonction des opportunités économiques ou des contraintes politiques, ce qui a créé un maillage de relations familiales, de combinaisons ethniques et religieuses complexes, sur fond d'origine arabe ou persane, et d'obédience chiite ou sunnite. Dans la mesure où ce substrat a fourni le socle à des migrations qui se poursuivent de nos jours, on peut considérer que ces mouvements participent des migrations provoquées ou accrues par la richesse nouvelle de la rive sud. Comme le pouvoir politique des États du Conseil de Coopération du Golfe est aux mains de dynasties de souche bédouine, arabe et sunnite (à l'exception de l'Oman, dominé politiquement par la branche ibadite de l'islam), les éléments originaires de la rive nord sont soit considérés comme des citoyens de seconde catégorie, soit comme des immigrants culturellement proches, soit comme des "bidoun " , des apatrides dont l'origine est niée ou récusée, et dont les allégeances réelles sont indécises. Quoi qu'il en soit, l'immigration ou plutôt les migrations sont à l'origine du peuplement actuel du Golfe, et les conflits d'antériorité y sont encore virulents, comme source de légitimité de pouvoir ou de citoyenneté: monarchies arabes originaires du cœur de la péninsule, paysanneries oasiennes arabes chiites, pêcheurs et prolétaires d'origine persane, grande bourgeoisie marchande d'origine tantôt arabe sunnite, tantôt persane chiite, sans oublier les descendants d'esclaves africains qui formaient parfois jusqu'à la moitié de la population (Montigny, $2002)^{6}$. Certes, ces minorités ont souvent la nationalité du pays de résidence, où elles sont installées depuis plusieurs générations; mais leur citoyenneté n'est pas aussi complète que celle des citoyens d'origine bédouine, arabe et sunnite, et leur accès à la redistribution de la rente pétrolière est amputé de manière variable (Montigny, 1996). Ces migrations oscillent toujours selon le pendage des conditions les plus favorables: c'est ainsi que depuis la Révolution islamique iranienne en 1979, de nombreux hommes d'affaires, venus cette fois-ci des grands centres urbains du nord de l'Iran, ont choisi de s'installer à Doubaï, devenue la fenêtre de l'Iran sur le monde extérieur ${ }^{7}$. Cette communauté joue désormais un rôle de premier plan dans la vie économique de l'Emirat, où elle a détrôné les grands commerçants d'origine indienne installés depuis le début du XX ${ }^{\text {ème }}$ siècle. 
$6 \quad$ Les cadres politiques modernes ne rendent pas compte de la nature et de l'intensité des relations régionales ou locales antérieures au découpage des frontières; c'est ainsi que le Golfe a des relations humaines particulièrement étroites avec le Baloutchistan, au point que celui-ci constitue l'une des dimensions majeures de son héritage historique et de sa palette humaine actuelle. Ce territoire, partagé entre Iran, Afghanistan et Pakistan, porte le nom du peuple musulman sunnite qui l'habite et est lui-même subdivisé en sous-ensembles régionaux comme le Makran ; il occupe le rivage iranien du détroit d'Ormuz et pakistanais de la mer d'Oman, où Oman possédait l'enclave de Gwadar, rétrocédée en 1958 au Pakistan.

7 Les Baloutches, à l'origine mercenaires et pêcheurs, ont fait souche dans les villages côtiers de l'Oman, de Sour à la Bâtinah; ils forment encore l'ossature des forces de sécurité et de l'armée des différents émirats du Golfe, mais on les trouve aussi dans le commerce, la banque et dans les professions désormais réservées aux nationaux, comme chauffeurs de taxi. Détenteurs de la nationalité locale, ils ont néanmoins gardé leur langue, leurs traditions et leur mode de vie, plus ouvert que celle de leurs concitoyens de culture arabe. Les relations avec le pays d'origine demeurent vivaces, surtout pour les migrants les plus récents, qui conservent la nationalité d'origine: relations aériennes et maritimes témoignent de ces flux, tout comme la prospérité relative des vallées du Makran ou des rivages qui font face à l'Oman (Nicolini, 1998).

8 Les Baloutches occupent donc une situation intermédiaire entre la population qui se considère comme autochtone et les immigrants, entre son installation ancienne et son altérité ethno-linguitique par rapport à la population tribale de souche arabe. Citoyens des États où ils sont installés comme l'Oman, ils en portent l'habit national; mais ils sont reconnaissables à leur traits physiques, à leur accent et à leurs imperfections en arabe et leur intégration ne va pas de soi : avec l'intensification de la compétition entre jeunes nationaux pour l'emploi, leur dynamisme relatif et leur ouverture aux mœurs et aux comportements occidentaux excitent la jalousie des «vrais» Omanais, qui appellent à la remise en cause de leur statut national.

L'immigration arabe, ou la découverte de l'appartenance, parfois pesante, à la « nation arabe »

9 A la différence des précédents, les migrants venus du Levant, de la vallée du Nil, puis du Maghreb n'ont découvert les rivages du Golfe qu'à la faveur de la découverte du pétrole, dans l'immédiat après-guerre. Cette immigration était indispensable pour jeter les bases techniques et administratives de la modernité : l'entourage des princes, chargé de l'interface avec les compagnies et les puissances occidentales a été très tôt constitué d'hommes d'affaires, de juristes, de médecins et d'ingénieurs égyptiens ou libanais, qui ont édifié des fortunes colossales à l'ombre de leurs maitres. Le développement de l'éducation, de la santé, l'encadrement des chantiers, dans les villes comme sur les exploitations pétrolières n'auraient pas été possibles sans ces immigrés de toutes spécialités venus de Syrie, du Liban, de Palestine ${ }^{8}$ ou d'Égypte. Ainsi la mise en exploitation du pétrole du Koweït a coïncidé avec la nakba, « la catastrophe » de la fondation de l'État d'Israël en 1948: de nombreux cadres palestiniens chassés de leur foyer ont rejoint directement l'Émirat sous tutelle britannique, dont ils ont fait un État moderne, et dont ils constituaient $40 \%$ de la population jusqu'à l'invasion du pays par les forces irakiennes (Radi, 1993). Le Koweït était devenu pour eux une seconde patrie, soutenant financièrement et politiquement la lutte pour la libération de la Palestine (Hanafi, 1999). Ces premiers immigrants arabes des années cinquante et soixante 
apportaient avec eux un ferment de modernité et d'ouverture, voire de progressisme et de laïcité dans une région à l'écart des bouleversements que connaissait le MoyenOrient; ils y ont importé l'idée d'appartenance du Golfe à une nation arabe et des notions de changement politique, sous les labels du nassérisme, du baassisme ou de variantes du nationalisme arabe. Certains ont été à l'origine des premiers partis politiques de la région, d'autres ont rejoint même des mouvements révolutionnaires comme le Front Populaire de Libération de l'Oman et du Golfe Arabe (FPLOGA), qui ouvre un maquis marxiste-léniniste dans les montagnes reculées du Dhofar ${ }^{9}$ (Sonallah, 2002). En retour, ils allaient entrer par le biais de cette émigration en contact en contact avec le monde américanisé de la technologie, des méthodes de travail, des rapports hiérarchiques fondés sur la valeur et l'efficacité personnelles, différents des valeurs inculquées dans les écoles des nations européennes qu'ils avaient fréquenté dans leur pays d'origine ou en Europe même.

Cette première vague de cadres issus du Moyen-Orient allait être étoffée à partir des années soixante-dix par la vague de migrants aspirés par le boom pétrolier de 1973 : ceux-ci, souvent des jeunes originaires des campagnes les plus reculées et des banlieues misérables d'Égypte, de Jordanie, du Liban, de Palestine ou de Syrie ${ }^{10}$ allaient trouver dans le soudain besoin de main-d'œuvre du Golfe l'occasion d'échapper au chômage et à la misère. Le pactole qui se déverse soudainement sur le Golfe est converti en infrastructures de toutes sortes, en villes rasées ou qui s'étendent, édifiées de manière futuriste. Les chantiers qui s'ouvrent chaque jour font appel à une main-d'œuvre peu qualifiée, rendue disponible par l'échec des socialismes arabes et la libération économique à l'ordre du jour. Elle sera fascinée par l'alliance de la modernité technique offerte par les pétrodollars et de l'islam conservateur saoudien qui donne le ton dans la région, et ramènera ce nouveau système de valeurs, celui d'un islam « à l'américaine ", de retour au pays.

11 Les migrants originaires du Moyen-Orient seront donc, dans les années soixante et soixante-dix et quatre-vingt, par leurs économies placées dans les banques islamiques, puis par les idées qu'ils ramèneront de leur séjour dans le Golfe, parmi les principaux vecteurs d'un islamisme militant, soutenu par l'Arabie Saoudite et les autres émirats du Golfe.

12 Les réactions du monde arabe à l'invasion du Koweït en août 1990 montreront cependant que les transformations socio-économiques du Golfe, dont les migrants sont à la fois les témoins et les acteurs, sont à l'origine de sentiments ambigus, où l'engouement fait place à la jalousie et à la colère : les pays qui font mine de soutenir l'Irak dans son invasion du Koweït (la Palestine représentée par l'OLP de Yasser Arafat, la Jordanie, le Yémen, le Soudan) voient leurs ressortissants expulsés sans ménagements, souvent en abandonnant les économies de toute une vie. Pour des raisons de sécurité, les Égyptiens, comme avant eux les Algériens, sont écartés, de peur de soulèvements et de subversion interne.

13 Aujourd'hui la présence arabe subsiste, en particulier dans l'administration et dans l'enseignement, où un pays comme la Tunisie occupe désormais une place de choix aux côtés de l'Égypte ; certains pays ostracisés depuis 1991 voient les portes de l'émigration, vitales pour leur économie ${ }^{11}$, s'entrouvrir à nouveau. Mais aujourd'hui, la demande de main-d'œuvre originaire du monde arabe est durablement amoindrie, à la fois par la fin des grands chantiers d'équipement, la nécessité économique et sociale pour ces pays de former et de fournir un emploi à leur propre jeunesse, et par la méfiance persistante à 
l'égard de pays tenus en suspicion pour leurs sentiments de rancœur à l'égard des pétro-monarchies et d'exaltation religieuse à base de rejet de l'hypocrisie et de la corruption des puissants et des alliés de l'Occident. Les deux millions d'Égyptiens qui vivaient en Irak durant la guerre Iran-Irak, pour remplacer les hommes au front, n'y sont pas retournés, non plus que les Palestino-Jordaniens qui formaient les cadres des services de sécurité et de l'armée des différents émirats, et l'émigration n'est plus la panacée pour les pays dont la jeunesse, même diplômée, est majoritairement au chômage.

L'immigration asiatique, ou la symbiose du Golfe et du sous-continent indien, clés de l'ouverture à la mondialisation

14 Les migrants venus du Moyen-Orient cèdent la place, pour les emplois les moins qualifiés surtout, à une main-d'œuvre originaire d'Asie du Sud et du Sud-est. A vrai dire, la présence de communautés asiatiques implantées sur les rives du Golfe n'est pas nouvelle. Les relations commerciales, facilitées par les vents de mousson, ont été popularisées par les récits de Sindbad le marin, héros mythique de l'Irak abbasside du $\mathrm{VIII}^{\mathrm{ème}}$ siècle. Mais c'est la mainmise britannique sur la région à partir du xix ${ }^{\mathrm{ème}}$ siècle qui favorisa l'implantation de sujets indiens de la Couronne, d'autant que le Golfe fut placé dans l'orbite du Raj, avec Bombay comme pôle économique et la roupie pour monnaie. Ces membres des communautés marchandes, numériquement faibles, sont économiquement très puissants ; ils sont surtout implantés à l'orée du Golfe, en Oman et à Doubaï. Ils sont originaires des provinces du nord-est de l'Inde, Bombay, le Gudjerat et le Sind, et sont de confession soit hindoue (les Bunya ou Banians) ou chiite (les Khodja ou Lawatiya originaires initialement d'Iran). Autrefois financiers et créanciers des émirs, à la tête d'empires commerciaux monopolistiques, ils ne se sont pas mélangés avec le reste de la population, et vivent encore parfois, comme les Lawatiya de Moutrah en Oman, dans des quartiers à part, fermés aux intrus. Considérés comme des agents du colonialisme britannique, ils furent longtemps jalousés, voire détestés ; si aujourd'hui de nombreux Indiens musulmans implantés de longue date ont acquis la nationalité de leur pays d'accueil, leur loyauté à l'égard de celui-ci demeure sujette à caution, et ils gardent prudemment d'étroites attaches avec l'Inde.

Si des courants migratoires ont drainé dès la découverte des hydrocarbures, les ruraux du bassin de l'Indus pakistanais et des vallées afghanes, comme les ouvriers du Gujerat vers les chantiers du Golfe, ce n'est qu'à partir des années quatre-vingt que se déclenchent les grandes vagues de migration asiatique qui vont littéralement submerger le Golfe après la première guerre du Golfe, pour remplacer les migrants arabes devenus indésirables. Selon une étude de l'OIT, plus de $20 \%$ des migrants dans le Golfe en 1995 étaient Indiens (3,6 M officiellement, et en fait déjà plus de 4 millions) : leur nombre était passé de 154000 en 1975, à 600000 en 1981 et 1,15 M en 1992. Mais leur proportion s'élève d'ouest en est, suivant en cela l'ancienneté et la densité des relations entre les pays de départ et d'accueil, c'est-à-dire selon une proximité culturelle qui rend leur intégration plus facile. Ainsi les Indiens constituent-ils $42 \%$ des migrants aux Émirats Arabes Unis (la population indo-pakistanaise y représentait déjà en 1996 près de $60 \%$ de la population, contre moins de $20 \%$ de nationaux); en revanche, à Bahreïn, les nationaux représentent $63 \%$ du total contre $13 \%$ d'Asiatiques, $1 \%$ d'immigrants arabes et $8 \%$ d'Iraniens, selon des estimations pour $1999^{12}$. Mais si l'on ne considère que la tranche d'âge de 15 à 64 ans, la proportion d'étrangers s'élève à $44 \%$. Au Qatar en revanche (550 000 habitants), les nationaux ne représentaient déjà pas plus de $20 \%$ de la population, contre $36 \%$ pour les Asiatiques, avant le boom 
provoqué depuis deux ans par la mise en chantier de l'énorme gisement de gaz du North Field : l'afflux de main-d'œuvre y est considérable et marginalise encore plus la population autochtone ${ }^{13}$.

16 Au total, un million de migrants partent chaque année du sous-continent indien en direction du Moyen-Orient ; les Indiens, largement majoritaires, ne sont cependant pas les seuls, puisque le Golfe emploie environ 400000 Népalais et 155000 Sri Lankais.

17 Les foyers d'émigration sont très localisés : sur plus d'un million d'Indiens travaillant aux Émirats Arabes Unis, la moitié provient du Kerala. Parmi les 33 millions d'habitants de cet État, 3,75 millions vivaient à l'extérieur en 1998, dont deux millions à l'étranger. Ils faisaient vivre 7 à 8 millions de personnes restées au Kerala ${ }^{14}$. À l'intérieur même de l'État, la propension à émigrer varie grandement selon les régions, et, si l'Arabie Saoudite attire $40 \%$ des partants, suivie par les Émirats Arabes Unis, les destinations préférentielles varient considérablement d'un district, voire d'un village à l'autre.

Les spécialisations professionnelles et nationales

18 Contrairement à une vision simplificatrice assez répandue, il n'y a pas dans le Golfe d'assignation automatique des postes occupés en fonction de la nationalité. Les diplômes, les compétences et les inclinations personnelles permettent théoriquement à chacun, en fonction de la demande du marché et des conditions de son recrutement, de trouver un emploi dans le domaine de son choix, quelle que soit sa nationalité d'origine ${ }^{15}$. Il y a cependant, bien entendu, des secteurs où telle ou telle communauté est majoritaire : ainsi dans l'ensemble du Golfe, en 1996, le tiers de la main-d'œuvre indienne, soit 425000 personnes, était employée dans le bâtiment, qui est le principal secteur d'emploi de cette communauté, avec le commerce de détail.

19 L'emploi sur les grands chantiers d'infrastructures, d'équipement industriel ou de construction est régi de façon rigoureuse : les ouvriers sont embauchés dans le pays d'origine par des intermédiaires, pour la durée du contrat et se voient retirer leur passeport pour la durée du séjour. Ils sont logés dans des camps ${ }^{16}$, dans des dortoirs collectifs et n'ont pas de contact avec le pays d'accueil. À la fin du chantier, ils sont rapatriés collectivement dans leur pays. Dans cette catégorie, outre les migrants en provenance du sous-continent indien, on trouve de nombreux Coréens, Thaïlandais, Philippins, les premiers souvent amenés par leur entreprise. Mais les gros chantiers se font plus rares, dans la mesure où l'équipement de ces pays a été réalisé au cours des vingt années écoulées, et les compétences et les origines de la main-d'œuvre se diversifient : depuis 1995, les Émirats Arabes Unis ne délivrent plus de visas pour des travailleurs sans qualification, et tout employeur d'un étranger doit verser une caution de 3000 Dh par an, suivant en cela la tendance générale qui est à la " nationalisation " des emplois qui peuvent être occupés par des employés locaux.

20 La qualification dépendant du niveau d'éducation, elle est souvent rapprochée de l'origine régionale: ainsi les montagnards baloutches, afghans ou originaires des provinces du Nord-Ouest du Pakistan sont-ils présents en grand nombre dans les travaux de force, les Bangladais, Sri lankais ou Philippins dans le nettoyage urbain, tandis que les Kéralites, qui ont un niveau d'éducation élevé et une bonne connaissance de l'anglais, sont majoritaires dans le commerce de détail ${ }^{17}$. Le marché du travail domestique, généralisé, se partage entre deux groupes féminins : les Sri Lankaises et les Philippines; mais les compétences réelles ou supposées des deux catégories sont estimées différemment, et si le salaire moyen d'une Sri Lankaise est de l'ordre de 150 \$ US par mois, celui d'une Philippine, qui a suivi une scolarité plus complète, maitrise 
mieux l'anglais - et est catholique - atteint plutôt 350 US $\$^{18}$. Les Philippines sont également majoritaires parmi le personnel hospitalier. Mais d'autres nationalités sont également représentées dans le travail domestique : Érythréennes, Mauriciennes, voire Seychelloises, comme au Liban ou en Jordanie.

Recrutement, précarité et exploitation de la main-d'œuvre

21 Le recrutement de ces migrants se fait pour l'essentiel à travers des agences spécialisées, dans un premier temps, dans la mesure où le travail dans les pays du CCG ne se fait qu'à travers un sponsor (kafil) qui sert de garant de l'étranger vis-à-vis des autorités ${ }^{19}$ Ces filières mettent en jeu des recruteurs du pays de départ, qui répondent à la demande d'organisateurs du «trafic » de main-d'œuvre, en fonction théoriquement des besoins du marché. Ces « importateurs » sont souvent des personnages en vue, qui monnayent ainsi leur position. En effet, le migrant se voit avancer son voyage, son visa et doit payer un droit pour le service qui lui est rendu. Souvent, le remboursement de ces avances, à des conditions usuraires, représente à lui seul une ou plusieurs années de labeur sur place.

Une fois arrivés, les migrants se voient confiés à leur kafil, qui garde leur passeport et les fait travailler pour son compte ou les place chez un employeur. En règle générale son prélèvement représente entre le tiers et la moitié des revenus de l'employé. Mais la gamme des conditions économiques et sociales est très vaste, entre la domestique "sponsorisée " par le maître de maison, et qui échappe à tout contrôle et à toute protection au boutiquier qui se voit confier la gérance d'un magasin. Pour les cadres, fonctionnaires et a fortiori hommes d'affaires étrangers, la situation est tout autre: leur contrat est négocié de gré à gré, ils ont le droit de s'installer, de faire venir leur famille ${ }^{20} .$. Mais tous sont à la merci de leur employeur : ils peuvent être renvoyés sur le champ, n'ont aucune protection sociale ou syndicale, et leur renvoi signifie le retour définitif au pays et une catastrophe par la parentèle, souvent nombreuse, qui dépend de leurs envois.

L'exploitation dont ils sont l'objet peut dégénérer en véritables abus ; ainsi des émeutes ont éclaté en novembre 1999 au Koweït, des milliers d'Égyptiens protestant contre leur cantonnement: ils avaient été importés sous le couvert d'un Ministre qui avait empoché sa part de l'opération, mais les emplois attendus n'existaient pas. Les pays d'origine ont des politiques diverses face au phénomène de l'émigration : l'Inde a mis en place une politique de formation et de soutien de ses migrants (création en 1978 d'une Overseas Manpower Corporation, suivie de l'Emigration Act en 1983), tandis que le Sri Lanka laisse ses ressortissants livrés à la rapacité et aux exactions des employeurs ${ }^{21}$, bien que les remises des émigrés au Moyen-Orient y constituent le quatrième poste de recettes étrangères, après la confection, le tourisme et le thé.

L'exception du Golfe : le refus de l'implantation des migrants

L'immigration dans le Golfe se caractérise par une grande précarité individuelle, avec la quasi-impossibilité de s'implanter durablement dans le pays et d'y faire souche. En effet, les naturalisations, qui ne sont le fait que du Prince, sont rarissimes; la nationalité ne se transmet que par le sang, transmis par le père. Il arrive même que les enfants d'une mère étrangère ne puissent pas bénéficier de la nationalité du père, et que la citoyenneté des nationaux de confession chiite soit contestée (Montigny, 1996). Certes, les jeunes travailleurs venus sans leur famille ne songent guère à s'implanter ${ }^{22}$, mais de nombreuses familles originaires en majorité du Moyen-Orient sont installées depuis trois générations et considèrent désormais leur pays de résidence comme une 
patrie d'adoption. Ils revendiquent désormais discrètement sinon l'intégration, du moins une possibilité d'implantation stabilisée par l'accès à l'immobilier, un titre de résidence plus durable, voire une possibilité de participer aux choix stratégiques d'un émirat comme Doubaï (El Ghoul, 2003). Cette intégration économique et sociale est défendue par certains responsables, qui considèrent qu'elle donnerait une certaine consistance humaine à des États qui paraissent parfois construits sur le sable, et dont la fragilité a été démontrée lors de l'invasion du Koweï. Mais elle se heurte à l'angoisse des nationaux à la perspective d'être marginalisés par les immigrants ${ }^{23}$.

L'heure est dans tout le Golfe, on l'a vu, à la «nationalisation » des emplois. L'objectif de ces politiques est double: procurer des emplois aux nouvelles générations, que la distribution de la rente ne suffit plus à entretenir et garder sur place les devises que les immigrés expédient dans leur pays. Dans le débat en cours actuellement autour de l'» omanisation" des emplois, l'argument de ses partisans est que, même si les travailleurs omanais doivent être payés plus cher pour un travail moindre que leurs homologues importés, leur salaire demeure dans le pays, où il est consommé et investi, ce qui est un gain pour l'économie nationale. En fait, il paraît illusoire d'envisager de se passer de la main-d'œuvre importée, tant la culture rentière a fait de ravages dans les mentalités, dans le Golfe comme à un moindre degré dans l'ensemble du Moyen-Orient arabe.

L'impact économique multiforme des migrants

On constate en outre que les migrants sont aussi des intervenants actifs dans l'économie du Golfe, puisqu'ils consacrent une part importante de leurs économies à acquérir des biens sur place pour les rapporter au pays, pour leurs propres besoins ou pour la revente. Ils participent ainsi activement au commerce de réexportation dont Doubaï, par exemple, s'est fait une spécialité. Ressentis comme une menace pour l'homogénéité sociale, les migrants sont donc indispensables au fonctionnement de l'économie. Et les mesures prises pour en réduire le nombre sont donc contreproductives ou bien répondent à d'autres objectifs, comme le souci de réguler un volant de clandestins, qui, moderne "armée de réserve du Capital ", maintienne bas le prix de la main-d'œuvre. L'Arabie Saoudite expulse ainsi 350 à 450000 immigrants illégaux par an ; mais quand, comme à l'automne 1996, les autorités des E.A.U. organisent une grande opération d'expulsion de 167000 immigrants illégaux, celle-ci pose des problèmes inattendus : elle entraîne une baisse de la population de $7 \%$, donc une baisse de la consommation et une hausse du coût de la main-d'œuvre de $40 \%$, car le sponsorship de la main-d'œuvre légale de remplacement revient à $5000 \mathrm{Dh}$ par an: la hausse des prix dans les secteurs de l'hôtellerie ou le bâtiment atteint de 10 à $20 \%^{24}$.

Les migrants ont aussi un impact irremplaçable sur les sociétés de départ : au Kerala, la proportion de la population vivant en dessous du seuil de pauvreté est passée en dessous de $12 \%$. Le nombre de chômeurs a baissé de plus de $30 \%$. En 2000, les remises représentaient $23 \%$ du PIB et dix fois les subventions du gouvernement central, quatorze fois les recettes dues à l'industrie de la pêche et vingt fois celles dues aux épices, les deux principaux postes d'exportation de l'État. L'émigration y est considérée comme une forme non-conventionnelle de développement, mais qui a pour avantages de ne pas provoquer d'effets nocifs sur l'environnement, de ne pas requérir d'investissements en capital, même si la concurrence qui règne sur le marché des migrants est forte: c'est pourquoi l'éducation est le moyen de maintenir la maind'œuvre « exportable » à un niveau de qualité compétitif. 
Une imbrication socio-culturelle de plus en plus prononcée entre Golfe et souscontinent indien

En définitive, les indicateurs économiques ci-dessus ne sont-ils pas que l'expression quantifiée d'impacts plus profonds et de l'imbrication indélébile des modes de vie et des cultures de ces deux ensembles régionaux, Golfe d'un côté, sous-continent indien de l'autre? Il est encore difficile de répondre avec précision à cette question, et l'on doit se contenter d'observations empiriques :

- la langue arabe est sans doute devancée dans le Golfe par l'ourdou, le malayalam (langue du Kerala), le hindi et peut-être le persan. Mais les autochtones, minoritaires, n'emploient-ils pas eux-mêmes en arabe des mots et des locutions empruntées à ces langues? Il serait intéressant que des linguistes se penchent sur l'existence éventuelle d'un sabir composé d'un cocktail des langues du Golfe, d'une évolution éventuelle vers une lingua franca où l'arabe et l'anglais auraient certes une place prépondérante, mais où transparaîtraient les dynamismes respectifs des différentes communautés linguistiques en contact. Il ne faut pas négliger l'impact, non seulement de la conversation courante, mais de la réception dans chaque foyer des chaînes de télévision du sous-continent, tandis que les journaux anglophones, majoritaires, donnent la priorité aux nouvelles d'Asie, faisant vivre leurs lecteurs, qui ne sont pas tous originaires du sous-continent, à l'heure de Mumbaï (Bombay) ou de Chennaï (Madras) ;

30 - les goûts, les modes, les valeurs et les comportements sont également très influencés par la présence des communautés d'immigrés, sans que l'on sache parfois de quand date l'adoption de telle recette de cuisine, de tel mode musical, de telle coutume vestimentaires, ni ce qu'elle représente comme acculturation réciproque, pour des sociétés réputées, des deux côtés, pour être très conservatrices dans leurs attitudes sociales et culturelles.

Un système social fondé sur une ségrégation ethnique dangereuse

31 Finalement, le maintien d'une ségrégation stricte entre nationaux et étrangers, ce refus d'intégrer l'Autre, de lui faire une place alors même que tant d'affinités tissées au fil des siècles rapprochent les sociétés qui entourent le Golfe, cette ségrégation née de la richesse, et qui vise à protéger les oisifs de la concupiscence de ceux qui transforment cette richesse en confort, n'est-elle pas une innovation artificielle? N’y a-t-il pas là, avant tout, le remplacement des clivages sociaux antérieurs, au sein des tribus comme des groupes de pêcheurs de perles ou de marchands, par une stratification ethnique garante de la concentration de la rente par quelques oisifs réputés inoffensifs ? Cet ordre n'est pas neutre et répond à la volonté des Puissances qui, en dernière instance, contrôlent le destin de cette région : la Grande-Bretagne à l'origine, et aujourd'hui les Etats-Unis, attentifs à garder la richesse pétrolière sous un contrôle étroit, et à éviter qu'elle ne soit répartie d'une manière plus efficace en faveur d'un développement menaçant pour leur domination. La nationalité, concept importé, a certes remplacé ou du moins recouvert - les statuts qui régissaient les sociétés locales d'avant le pétrole : les tribus se distinguaient par la noblesse de leur généalogie et les activités dont elles tiraient leur subsistance, les tribus nobles des tribus soumises, les hommes libres des esclaves, etc. Mais l'existence de multiples traits culturels communs, la facilité avec laquelle sont échangés de nouveaux éléments montrent l'arbitraire des nouvelles barrières statutaires mises en place. 
peut se demander combien de temps cette résistance, fondée sur le système de la kafala et sur le jus solis, pourra durer face aux pressions qui s'exercent sur ce ventre mou trop riche et trop faible qu'est le Golfe. D'autant que de nouvelles formes de solidarité ou de clivage peuvent aisément y être activées, comme les instances religieuses qui peuvent rapprocher les Musulmans nantis autochtones de leurs coreligionnaires des pays environnants en proie à la misère ou à la violence, comme le montrent les événements qui se déroulent actuellement dans cet arc de crise. Le Golfe peut aisément devenir la caisse de résonance de tous les conflits de la région, et sa diversité ethnique provoquer une explosion fatale ${ }^{25}$, si un assouplissement n'est pas introduit dans son fonctionnement social.

\section{BIBLIOGRAPHIE}

EL-GHOUL Bernard (2003) De la cité marchande à la cité globale. Pouvoir et société à Doubaï, Thèse, Institut d'études Politiques de Paris.

HANAFI Sari (1999) Insertion économique et marginalité sociale in Entrepreneurs dans le monde arabe, Les Cahiers de l'Orient n ${ }^{\circ} 55,3^{\mathrm{ème}}$ tr., pp. 133-145.

MONTIGNY Anie (1996) Les Arabes de l'autre rive, Cahiers d'études sur la Méditerranée orientale et le monde turco-iranien, $\mathrm{n}^{\circ} 22$, juillet-décembre, pp. 51-83.

MONTIGNY Anie (2002) L'Afrique oubliée des noirs du Qatar, Journal des Africanistes, 72 (2), pp. 213-225.

NICOLINI Béatrice (1998) International Trade Networks : the Oman enclave of Gwadar, communication à la Oman Tagung, Bonn, 20 juillet 1998.

RADI Lamia (1993) Les Palestiniens du Koweït en Jordanie : confrontation ou intégration à la société d'accueil ? Mémoire de DEA, Institut d'études Politiques de Paris, 1993, 87 pages.

SONALLAH Ibrahim (2002) Warda, trad. franç. R. Jacquemond, Actes Sud.

\section{NOTES}

1. -A noter que Bahreïn et Oman sont des exportateurs traditionnels de main-d'œuvre, et que leurs faibles ressources en hydrocarbures les conduisent à envisager une reprise des migrations en direction des Etats voisins plus riches au sein du Conseil de Coopération du Golfe : Bahreïn en direction du Qatar, Oman en direction d'Abou Dhabi. 2. -Charjah, Ajman, Umm el-Qaïwaïn, Ras el-Khaïmah et Fujairah, membres de la fédération des Émirats Arabes Unis.

3. -Sauf pour certaines fonctions comme les instituteurs de l'enseignement primaire, les petits boutiquiers de village ou les ouvriers agricoles dans les palmeraies.

4. -Au XIX siècle, le Résident politique britannique pour le Golfe était installé à Boushehr, sur la rive nord, et un port comme Doubaï n'a dû sa fortune qu'au déclin du sultanat arabe de Lingeh, qui lui faisait face sur la rive persane, au début du $\mathrm{XX}^{\mathrm{e}}$ siècle. 
L'expression même de golfe «persique » aujourd'hui décriée par les Arabes, exprime bien cette domination séculaire de la rive nord.

5. -Au sens littéral les « sans » en arabe. Ils seraient 120000 au Koweït, d'origine bédouine ou paysanne du Bas-Irak, mais on les retrouve à Bahreïn, où ils sont supposés d'origine iranienne.

6. -Voir en particulier l'analyse du statut social des communautés Huwalah et Baharnah au Qatar. Sans être considérés comme immigrés, ces groupes qui jouissent de la nationalité qatarienne et jouent un rôle important dans le commerce et les affaires sont stigmatisés pour leur non-appartenance à une tribu arabe, et pour leur origine de la rive nord du Golfe (les Baharnah sont chiites).

7. -On estime à 2000 le nombre des maisons de commerce iraniennes ayant transféré leur siège à Doubaï.

8. -Voir les travaux de Sari Hanafi sur les entrepreneurs palestiniens dans le Golfe.

9. -Ibrahim Sonallah a publié une relation romancée de cette expérience, Warda, traduit en français.

10. -Auxquels il faut ajouter près d'un million de Yéménites en Arabie Saoudite.

11. -Dans les années soixante-dix, plus de $30 \%$ de la population active jordanienne travaillait dans le Golfe.

12. -630000 habitants dont 227000 étrangers ; mais cette faiblesse numérique de la population immigrée est, dans ce cas précis, largement due à la pauvreté relative de l'île, qui ne constitue plus depuis plusieurs décennies un État rentier, et où le chômage (15\%) frappe une part importante de la jeunesse.

13. -Sans compter le cas bien particulier des dizaines de milliers de militaires américains qui y ont établi leur quartier général opérationnel pour le Golfe et l'Irak (base d'Al-Udeid, dans le sud du pays).

14. -A titre d'indication sur le rythme de progression du phénomène migratoire, le nombre de départs du Kerala est passé de 11679 en 1980 à 89542 en 1990 et 155726 en 1997.

15. -Bien souvent cependant, quelques soient les promesses faites au départ, le sponsor impose ses exigences sur place et affecte l'employé importé à un poste à pourvoir, indépendamment de ses qualifications et de ses souhaits éventuels.

16. -Ce serait le cas de $32 \%$ des migrants indiens dans le Golfe (The Hindu, 26 janvier 2002).

17. -L'État du Kerala considère l'émigration comme une ressource-clé du développement et suit une politique d'éducation qui vise à améliorer constamment les qualifications de sa main-d'œuvre ayant vocation à l'exportation.

18. -La nationalité des domestiques est ainsi un marqueur social de leur employeur. 19. -Les autres modes d'entrée illégaux sont l'immigration clandestine, par bateau, depuis les côtes d'Iran ou du Pakistan, mais surtout pour l'Arabie Saoudite le maintien sur place des voyageurs musulmans détenteurs d'un visa de pèlerin.

20. -A Doubaï, un revenu mensuel minimum de 4000 Dh est exigé pour obtenir le droit de faire venir sa famille.

21. -Scandale en 2000 du consulat de Sri Lanka en Jordanie : le consul accouchait clandestinement à sa résidence les dizaines de ressortissantes enceintes après leur viol par leur employeur, et alimentait un lucratif trafic d'adoption avec les nouveaux-nés confisqués à leur mère.

22. -Au Kerala, on estime le nombre de couples séparés par l'émigration d'un des membres à un demi-million (The Hindu, 26 jan 2002). 
23. -A Doubaï vient d'être créée une Association de citoyens réclamant l'arrêt de l'immigration et par conséquent aussi la fin des stratégies d'ouverture et de développement économique pour laquelle les immigrés sont indispensables, considérant que la population autochtone ne représenterait pas plus de $4 \%$ de la population totale de l'Emirat.

24. - "Labour exodus takes the edge off expansion ", Middle East Economic Digest, 6 décembre 1996, pp. 37-38.

25. -Dont les émeutes entre Hindous et Musulmans indiens à Doubaï en 1995 lors de la destruction de la mosquée Babri Masjid en Inde donnent un avant-goût.

\section{AUTEUR}

\section{MARC LAVERGNE}

Chargé de recherches au CNRS, Laboratoire Géotropiques, Université Paris X Nanterre, Bât. D, 3e étage, 200 avenue de la République, 92001 Nanterre cedex 